\title{
The Analysis of 220kV Transformer Abnormal DC Resistance
}

\author{
Lan Wei, Cheng Pengfei, Wang Qinghao, Liu Chenyang, Zhang Hua, Liu Bo, Li \\ Bo, Liu Chuanbo, Wu Yunguo, Huang Zijun, Dong Pin, Liu Zhaohui, Gao Wei \\ Fushun Power Supply Company, Liaoning Electric Power Company Limited, State Grid, China, \\ fushunpowersupply@163.com
}

Keyword: wiring board bolts loose, coefficient of expansion, metal deformation, high temperature disk spring

\begin{abstract}
At the main transformer for Helping on the 1st pre-test and found that the 1st variant of $66 \mathrm{kV}$ side of the A-phase winding DC resistance is too large, and the phase imbalance difference of $7.21 \%$. On all aspects of the test wiring, testing and other parts were examined and treated, the test data did not change, the maintenance personnel to open 66kVA transformer phase "General cap" and found four phase winding A first end of the conductive rod (wiring board) connection some bolts loose, re-tighten again retest DC resistance test data qualified.
\end{abstract}

\section{Introduction}

Helping substation 1st Main transformer on the 1st January 8, 2000 and put into operation, operation life for 13 years. Production of transformers Type: SFPSZ11-120000 / 220. Before 2013 blackout overhaul, all joints of the external transformer infrared thermal imaging tests. Found in the test phase $66 \mathrm{kV}$ side A higher temperature of both ends of the sleeve $26.2 \mathrm{C}$ (see Figure 1), the current 1000A; temperature while it is on the left side end portion of the sleeve relative to B $15.9 \mathrm{C}$, current $990 \mathrm{~A}$; ambient temperature $12.6 \mathrm{C}$. After a review of the recent transformer insulating oil chromatographic analysis report, the comprehensive analysis in accordance with the current load, ambient temperature, etc., we will not affect the safe operation of the transformer, and then a treatment can wait until the maintenance outage[1-4].

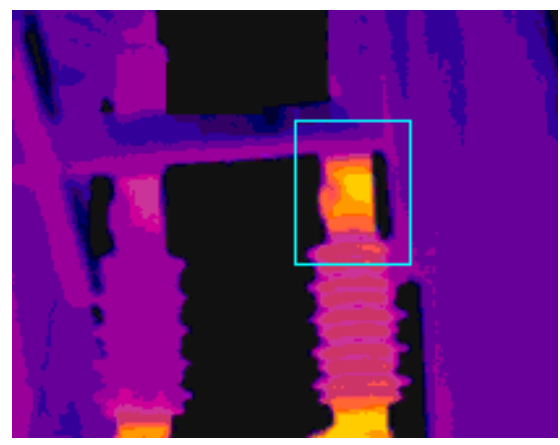

Figure $166 \mathrm{kV}$ of the A-phase infrared test tube case

April 23, 2013, in a power outage when the pre-trial, found on the 1st main transformer $66 \mathrm{kV}$ side of the A-phase winding DC resistance is too large. Three-phase data for Am0: 11.60 $\mathrm{m} \Omega, \mathrm{Bm} 0: 10.80 \mathrm{~m} \Omega, \mathrm{Cm} 0: 10.89 \mathrm{~m} \Omega$, by calculating the difference between the phase imbalances of $7.21 \%$, exceeding the "Regulations" provides only $2 \%$. Although we had a lot of consideration and treatment, but the test data remains unchanged after the analysis that is due to internal reasons transformer[5].

By maintenance personnel will be on the 1st transformer main transformer insulating oil put to $66 \mathrm{kV}$ side manhole below, open manholes, found the first phase winding A conductive terminal and connecting rod (wiring board) 4 bolts and some loose (see Figure 2) . 


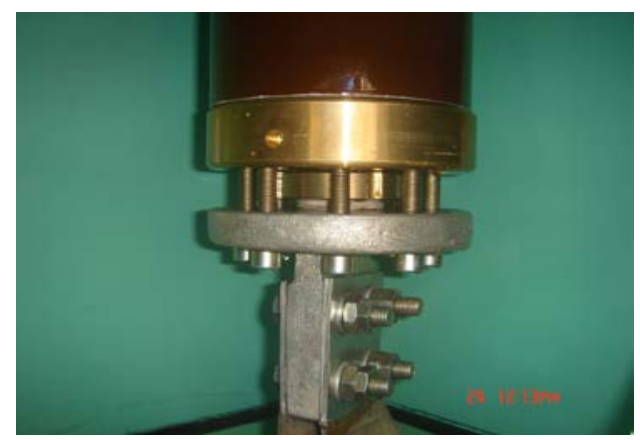

Figure 2 The 4 bolts loosening phenomenon

After re-tighten, try again side DC resistance test data $\mathrm{Am} 0: 10.56 \mathrm{~m} \Omega, \mathrm{Bm} 0: 10.58 \mathrm{~m} \Omega$, $\mathrm{Cm} 0: 10.52 \mathrm{~m} \Omega$, three-phase average difference of $0.56 \%$, and in accordance with the test data of qualified "rules" requirement. The following line of metal materials from the factory expansion and production processes, analysis of these issues, identify where the problem is put forward possible solutions to prevent the problem from happening again.

\section{Cause Analysis}

The cause of $66 \mathrm{kV}$ side of the A-phase transformer winding DC resistance is too large, is due to manufacturers in the production of the winding uneven solder when soldering the first end of the connector, and not using a dedicated thermostat tire tool intensify welded parts, warming to the tin when the melting point temperature of the extruder $232 \mathrm{C}$ excess solder to form the solder layer is not formed and the cavity between. When the first connector and the conductive rod end windings (wiring board) connection is used under the nut flat washer, rather than high temperature Disk Spring (not opening a spring washer, shape UFO), tighten the connection in this case, can not completely overcome repeated deformation of metallic materials after thermal expansion, resulting in the gradual relaxation bolt preload, thereby causing the DC resistance of the windings increases, the range of values deviate qualified lead winding DC resistance unbalance the difference exceeds the "regulations" provisions.

A different linear expansion coefficient $\alpha$ of the metal material is different. In a temperature range of $20 \sim 100 \mathrm{C}$ brass $\alpha=17.8 \times 10^{-6} / \mathrm{C}$, copper $\alpha=17.2 \times 10^{-6} / \mathrm{C}$, carbon $\alpha=10.6 \sim 12.2 \times 10^{-6} / \mathrm{C}$, tin $\alpha=2.3 \times 10^{-6} / \mathrm{C}$.

Since the wiring board is made of brass, copper winding material is winding first end connector portion sizes according to the design with multi-soldered copper, connecting them are four ordinary carbon steel bolts. The hardness of the order of each material used for steel "Brass> copper> tin, from the above data and the order is not difficult to see that the most vulnerable tin extrusion, and is not reversible.

With the assistance of the relevant operating personnel, the paper questions the transformer nearly five years of operation carried out inspection. That from 2005 to 2009 the annual maximum load, and maximum load transformer temperature from access to data. Specific figures is shown in Table 1

From Table 1 that, load September 18, 2006 the largest transformer, the highest temperature. Since this would mean that the highest temperature linear expansion of the metal material will be larger, and the transformer load with decreasing temperature, the line will follow the expansion of the metal material is reduced, the bolt preload will loose contact with the contact surface of the resulting the resistance becomes large. 
Table 1 Maximum load and transformer temperature

\begin{tabular}{c|c|c|c|c|c}
\hline DATA & $\begin{array}{c}2201 \\
\text { (A) }\end{array}$ & $\begin{array}{c}301 \mathrm{~A} \\
(\mathrm{~A})\end{array}$ & $\begin{array}{c}301 \mathrm{~B} \\
\text { (A) }\end{array}$ & $\begin{array}{c}201 \\
\text { (A) }\end{array}$ & Temperature(C) \\
\hline 2005.6 .21 & 208 & 388 & 946 & 312 & 54.6 \\
2006.9 .18 & 248 & 794 & 802 & 552 & 58.2 \\
2007.11 .29 & 156 & 576 & 452 & 900 & 54.8 \\
2008.7 .4 & 193 & 746 & 536 & 927 & 55 \\
2009.7 .28 & 146 & 409 & 554 & 633 & 55.1 \\
\hline
\end{tabular}

To help clarify the matter, according to information by Figure 2 qualitatively estimate the wiring board thickness is $200 \mathrm{~mm}$, winding first end two joint thickness $10 \mathrm{~mm}$ (not Solder layer thickness), the total thickness of the solder layer of about $2 \mathrm{~mm}$, only to consider the length of the bolt is in line expansion values of $212 \mathrm{~mm}$.

Since the transformer is assembled in early November, heating date yet to come November 15 , therefore, when the ambient temperature transformer assembly should be around $10 \mathrm{C}$, rather than the factory test reports record $21 \mathrm{C}$. When injected because the transformer insulating oil factory test according to the technical requirements is the result of warming, the calculation should not be substituted into the factory test report records transformer temperature, ambient temperature and should be considered deemed to body temperature of the transformer. For ease of calculation, taking the ambient temperature $10 \mathrm{C}$, and regarded as the body temperature of the transformer. Maximum operating temperature of the transformer temperature taken September 18, 2006 of 58.2 C, by the following numerical calculation shows that the first end of the winding line expansion, wiring boards, solder layer, the bolts at this temperature.

Wiring board (brass) linear expansion $=17.8 \times 10^{-6} \times(58.2-10) \times 200=0.1716 \mathrm{~mm}$;

The first end of the coil connector (copper) linear expansion $=17.2 \times 10^{-6} \mathrm{x}(58.2-10)$ $\mathrm{x} 10=0.0083 \mathrm{~mm}$; Solder layer linear expansion $=2.3 \times 10^{-6} \mathrm{x}(58.2-10) \mathrm{x} 2=0.0002 \mathrm{~mm}$; Bolt (plain carbon steel) linear expansion $=11.00 \times 10^{-6} \times(58.2-10) \times 212=0.1124 \mathrm{~mm}$; Difference in linear expansion $=0.1716+0.0083+0.0002-0.1124=0.0677 \mathrm{~mm}$.

Through the above analysis, the calculation shows that this part of the pressing force generated by the difference in pressure only by deforming the solder layer, this part of the expansion to offset the "space", such deformation is reversible. At the same time due to the ferromagnetic transformer runtime generated shock will have some of these parts of the shaking force and friction, long time is also easy to make the solder layer deformation accelerated, so that the bolt preload relaxation.

\section{Measures}

Manufacturers recommend improvements in the process. According to design requirements $66 \mathrm{kV}$ transformer winding between the first end of the multi-layer copper immersion tin after treatment, the use of tires with the winding temperature first end fixed to intensify, heated to $232 \mathrm{C}$ solder melts, then the appropriate intensify tire tool, extrusion winding first end of excess solder between the multi-layer copper is removed between the tip surface of the shaped cavity is formed (Figure 3),

Welding surface of the solder between the thin and uniform. Avoid sudden cooling, because the thermal expansion coefficient of the metal, the thermal conductivity of the different surface quality of the weld and will, cause the first end of the volume resistivity of the windings increases. To natural cooling, the temperature dropped to ambient temperature, and then releases the tire tool; winding first end fitting after such treatment, in operation issues mentioned in the text will not appear. 


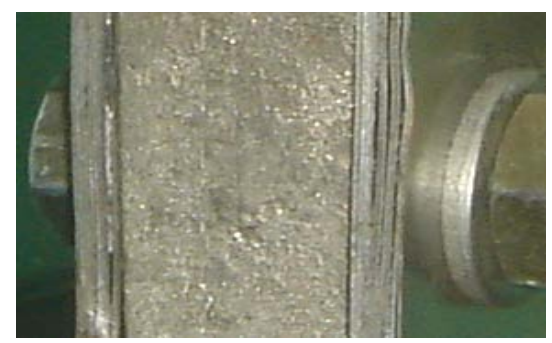

Figure 3 a multi-layer winding of the first end of the copper

End of the first winding welding can also choose to welding, arc welding, oxy - acetylene welding, electron beam welding, brazing, etc., from welding should be preferred to compare the effect of welding, and now most of transformer manufacturers chose argon arc welding technology. The manufacturer may recommend the use of TIG welding technology, but also solve the problem of effective one effective means.

For a similar case when a transformer during overhaul, transformer maintenance personnel should be added under high temperature Disk Spring nut and difficult plus flat washer, which not only solved the vibration caused by ferromagnetic first end fitting bolt preload transformer winding runtime relaxation; also solved due to the volume change of the metal due to repeated thermal expansion and pressure changes in the two contact surfaces, the contact resistance of the winding to ensure that the first end of the connector and the wiring board between the large change does not occur.

Do not add the opening spring washer on the flat washers. Due to the opening of the spring washer outer diameter smaller than the effective outer diameter of the nut pressing, reducing the pressure receiving area of the two contacting surfaces, but also easy to be deformed flat washer. At higher temperatures, larger temperature difference between the repeated changes both metal fastening, use the opening spring washer effect is not very good, it is better to use only flat washer's effect. However, when used flat washers pay attention to the press (edge burrs) facing up, tighten the nut flat washer after extrusion surface smooth, uniform force receiving surface to prevent the bolt preload relaxation but also add a drop Mother.

\section{Conclusion}

Some transformer malfunction, often appear on some tiny craft or because of improper choice of materials caused by loose bolts ascertain the reasons, but also to further improve the site analysis to determine the ability of our testing personnel issues; able to further enhance their professional and technical personnel transformer manufacturing plant in the small process requirements, so that the transformer can be a long-term safe and stable operation.

\section{References}

[1] Dongqiguo. Power transformer fault and diagnosis. China Electric Power Press, 2000.

[2] Chengdaxian. Mechanical Design Handbook. Chemical Industry Press, 1999.

[3] Zhonghongbi, Gaozhan-bang. Power transformer maintenance and testing manual. China Electric Force Press, 1999

[4] Han Aizhi. The Simple Method of Judging Transformer Winding Deformation. Transformer, 2003, 5(4): 14-16

[5] Chen Tianxiang, Wang Yinzhong. Electrical Test. China Power Press, 2010 